\title{
NEW OPTIMIZATION SCHEME FOR COOPERATIVE SPECTRUM SENSING TAKING DIFFERENT SNR IN COGNITIVE RADIO NETWORKS
}

\author{
Harsh N. Thakkar ${ }^{1}$, Kiran R. Parmar ${ }^{2}$ \\ ${ }^{I}$ M.E, Electronics \& communication dept, L D College of engineering, India \\ ${ }^{2}$ Professor, Electronics \& Communication dept, Govt. Engineering College, Gandhinagar, India
}

\begin{abstract}
This paper proposes new method to optimize the overall performance in hard cooperative spectrum sensing in cognitive radio. Optimization strategy is proposed in order to optimize the overall performance by variation of SNR. Here given strategy contributes to the methods in the literature by taking their performances to the peak point. Additionally, the effects of spectrum sensing technique type that used locally at each CR, the local SNR, and the total number of cooperated CRs on the optimal fusion rule are found. The energy detector (ED) spectrum sensing technique is examined as local spectrum sensing techniques. Here different error levels are founded by variation of SNR. The optimal number of CRs form minimizing the error at SNR 5,10,13,17,18,20 are found to be 4 or 5 , 5,5 or $6,6,8,9$ respectively.
\end{abstract}

Keywords: cognitive radio; spectrum sensing; cooperative spectrum sensing; cooperative spectrum sensing optimization

\section{INTRODUCTION}

Providing frequencies for the new wireless technologies increases the demand for spectrum, which is a scarce resource. An ineffective use of the already licensed spectrum, meets that high demand for the same [6].The technique of cognitive radio (CR), has been proposed to deal with such problems [11]. In Cognitive Radio system, the CR, which is called secondary user, senses its surrounding radio frequency (RF) environment to detect the vacant frequencies, which are being unused by their licensed users. These users are called primary users. Cognitive radio can use these vacant frequencies opportunistically to transmit and receive its data by adapting its transmission parameters like frequency. It enables secondary user/network to utilize the spectrum. So, it is the strategy proposed as a promising technology to improve spectrum utilization efficiency.

\section{EASE OF USE}

As defining the vacant frequencies is the way to exploit these unoccupied bands; the spectrum sensing is a key functional factor in cognitive radio. Energy detector (ED) is a one of the best spectrum sensing technique that does not require prior information about the Primary signal. This technique is simple, but that at the expense of its performance at low SNR. Cooperative spectrum sensing technique is proposed to eliminate the effects of shadowing and multipath fading on the spectrum sensing of primary user, when only one CR module is used [10]. In hard cooperation, each CR senses and decides about the $P R^{\text {"c }} \mathrm{s}$ signal in a specific frequency band, then a binary information 1 or 0 is sent to the CR base station(CR$\mathrm{BS}$ ) via dedicated control channel (CC), representing the presence or absence of Primary signal. Then, the CR-Base station decides on the all received digits using logical fusion rule. Different strategies and factors have been investigated to optimize the hard cooperative sensing performance by minimizing the total error probability, or maximizing the probability of detection [4]. It was achieved by optimizing the number of cooperated Cognitive radios and the threshold. The author has taken the global probability of detection in "OR" and "AND" fusion rules to peak by fixing the global false alarm probability In [7]. In [3] Strategies to decrease the total error probability under Neyman Pearson, and Bayesian criterions have been studied. In this paper, we add our contribution to the hard cooperative spectrum sensing optimization area, by adding an important factor that can be controlled in term to minimize the total error probability. Our work here can be applied to all mentioned optimization strategies to take them to the optimist point All optimization published works, focused only on ED as a local spectrum sensing. In this paper, the effects of using different numbers of CRs, different SNR on the optimal fusion rule have been investigated. The paper is organized as follows: Section III defines the models for the local spectrum sensing techniques when ED locally. Section IV presents the theoretical work of the cooperative spectrum sensing, that includes the optimization for the ED, and total number of CRs. Section V concludes the paper. 


\section{LOCAL SPECTRUM SENSING}

We have a number of $\mathrm{G}$ ( or $\mathrm{r}=1,2 \ldots G$ ) $\mathrm{CRs}$ in the $\mathrm{CR}$ network, where each CR performs spectrum sensing locally using Energy Detection. Each CR transceiver is supported by (N-IFFT/FFT) processers to perform both tasks of communication and sensing the environment. The primary transmitter with $\mathrm{N}$ subcarriers (N-IFFT/FFT) transmits OFDM-QPSK signal with energy over each sub carrier, and Ts which is symbol duration. So, each CR estimates the power within each subcarrier in the frequency domain, with $f_{i}=0$, $1 / \mathrm{N}, 2 / \mathrm{N}$. . . N-1/N are the bins of normalized frequency.

When we have fading environment where there are $P$ resolvable paths between the PR's transmitter and CR's receiver, $h_{p}, \mathrm{p}=0,1 \ldots \mathrm{P}-1$ represents the discrete - time channel impulse response between PR's transmitter and CR's receiver. The hypothesis test which is binary for CR spectrum sensing at the lth time is given by:

$$
\begin{gathered}
H_{0}: x_{t}(l)=w_{t}(l) \\
H_{1}: x_{T}(l)=\sum_{p=0}^{p-1} \quad h_{p} s_{t-p}(l)+w_{t}
\end{gathered}
$$

Where $1=0,1$. . . L- 1 is OFDM block's index, $x_{t}(l), w_{t}(l)$ and $s_{t}(l)$ denote the CR received, noise and PR transmitted samples. Additive white Gaussian noise with zero mean distorts the transmitted PR signal. The discrete frequency response of the channel is obtained by taking the $\mathrm{N}$ point FFT, with $\mathrm{N}>\mathrm{P}$ as given below:

$$
\mathrm{H}(\mathrm{fi})=\sum_{p=0}^{p-1} \quad h p e^{-j 2 \pi f i p}
$$

Here H0represents the absence of PR's signal and H1represents its presence. Now to evaluate performance of the local spectrum sensing using the $\mathrm{r}^{\text {th }} \mathrm{CR}$ user, the probability of detection Pd,r (fi), the probability of false alarm Pf,r (fi), and the probability of missed detection Pm,r (fi) at each frequency bin fi are considered based on the Neyman-Pearson (NP) criterion. The probability that the $\mathrm{r}^{\text {th }} \mathrm{CR}$ detector decides correctly the presence of the PR's signal is Pd,r (fi). The probability that the $\mathrm{r}^{\text {th }} \mathrm{CR}$ detector decides the PR's signal is present when it is absent is Pf,r (fi) . Lastly, is the probability that the $r^{\text {th }} \mathrm{CR}$ fails to detect the PR's signal when it is present is $\mathrm{Pm}, \mathrm{r}$ (fi).

As following the same work in [4], we assume that all CRs are much closed to each others in distances. Hence, wireless environments here can be assumed as an identical and independent in the $\mathrm{CR}^{\text {ee }} \mathrm{s}$ network, and SNR $=\frac{\|\left. H(f i)\right|^{2} E s}{\sigma^{2}}$ for each CR.
So, the Pd,r (fi), Pf,r (fi), and Pm,r (fi) will be replaced by $\mathrm{Pd}(\mathrm{fi}), \mathrm{Pf}(\mathrm{fi})$, and $\mathrm{Pm}$ (fi) respectively in the remaining part of this paper.

In General, the probabilities of detection $\mathrm{Pd}(\mathrm{fi})$, and false alarm Pd(fi) can be defined for normally distributed statistic as follow:

$$
\begin{aligned}
\mathrm{Pd}(\mathrm{fi}) & =\mathrm{P}(\mathrm{DEC}(\mathrm{fi})>\mathrm{y} / \mathrm{H} 1) \\
& =\mathrm{Q}\left(\frac{y-E[D E C(\text { fi }) / H 1}{\sqrt{V A R(D E C(f i) / H 1}}\right) \\
\operatorname{Pf}(\mathrm{fi}) & =\mathrm{P}(\mathrm{DEC}(\mathrm{fi})>\mathrm{y} / \mathrm{H} 0) \\
& =\mathrm{Q}\left(\frac{y-E[D E C(\text { fi }) / H 0}{\sqrt{V A R(D E C(f i) / H 0}}\right)
\end{aligned}
$$

Finally, the probability of missed detection $\mathrm{Pm}(\mathrm{fi})$ can be defined as:

$$
\begin{aligned}
\operatorname{Pm}(\mathrm{fi}) & =\mathrm{P}(\mathrm{DEC}(\mathrm{fi})<\mathrm{y} / \mathrm{H} 1) \\
= & 1-\mathrm{Q}\left(\frac{y-E[D E C(f i) / H 1}{\sqrt{\text { VAR(DEC }(f i) / H 1}}\right)
\end{aligned}
$$

Where DCE (fi), is the decision statistic at. The symbol $Q(x)$ is the complementary cumulative distribution function, $\mathrm{Q}(\mathrm{x})$ $=\frac{1}{2 \pi} \int_{x}^{\infty} e^{\frac{-t^{5} \mathrm{z}}{2}}$; it calculates the tail probability, and represents the threshold that we choose. Here we note that, y can be controlled based on L (threshold). Threshold's values are chosen based on $\sigma^{2}$.In this paper we examine the technique of local spectrum sensing at each $\mathrm{CR}$ receiver; the Energy Detector In the next two sub-sections a brief about technique is provided.

\subsection{Energy Detector}

In this scheme, the received signal is sampled to generate a finite discrete time samples series $\{\mathrm{xt} ; \mathrm{t}=0,1 \ldots \mathrm{N}-1\}$, where $t$ index of time. These samples are dot multiplied with rectangular window. Hence, for each frequency bin ,fi the decision statistic is computed by the summed energy over samples as:

$$
\operatorname{DECED}(\mathrm{fi})=\sum_{l=0}^{L-1}\left|\sum_{t=0}^{N-1} X t(l) e^{-j 2 \pi f i t}\right|^{\wedge} 2
$$

On the basis of central limit theorem, when L is large (e.g. L>10), the decision statistic can be approximated to normal distribution with the mean as given: 


$$
\begin{aligned}
\mathrm{E}[\mathrm{DECED}(\mathrm{fi})]=\mathrm{L} \sigma_{w}{ }^{\wedge} 2 & \text { for } \mathrm{H} 0 \\
=\mathrm{L}\left(|\mathrm{H}(\mathrm{fi})|^{\wedge} 2 \mathrm{Es}+\sigma_{w}{ }^{\wedge} 2\right. & \text { forH1 }
\end{aligned}
$$

and variance is defined as given:

$$
\begin{array}{ll}
\mathrm{E}[\mathrm{DECED}(\mathrm{fi})]=2 \mathrm{~L} \sigma_{w}{ }^{\wedge} 4 & \text { for H0 } \\
=2 \mathrm{~L} \sigma_{w}^{\wedge} 2\left(2|\mathrm{H}(\mathrm{fi})|^{\wedge} 2 \mathrm{Es}+\sigma_{w}^{\wedge} 2\right. & \text { for H1 }
\end{array}
$$

Here different local probabilities of ED-based spectrum sensing can be evaluated by substituting (7) and (8), into (3)(5).

\section{HARD COOPERATIVE SPECTRUM SENSING}

The spectrum sensing technique used locally at each CR has been described theoretically when ED is used. Generally, the cooperation in spectrum sensing is achieved when a number of CRs in the CR"s network send their local decisions to the CRBS via Communication Channel. Then after, CR-BS combines these decisions and decides finally about the presence of PR's signal in frequency bin fi. Here we note that, in our work, we are interested to examine the performance when the power spectrum is sensed at fi when the whole band under sensing is occupied by PR's signal in the case of H1. The hard cooperative spectrum sensing starts from performing local spectrum sensing using ED, The decision that $r^{\text {th }} C R$ makes is represented by binary digit $\mathrm{br}=$ ' 1 ', or H0represented by binary digit $b r=' 0$ ', based on its own local decision statistics. At the end, the CR-BS combines the received digits from different CRs to declare the final decision about the presence of primary signal.

All the received binary digits at the CR-BS from the different CRs, in the CR network, are fused together to declare the final decision using the logic rule as given below:

$$
\begin{aligned}
\operatorname{DECCOP}(\mathrm{fi}) & =\sum_{r=1}^{G} b_{r}>=\mathrm{g} & & \text { for } \mathrm{H} 1 \\
& =\sum_{r=1}^{G} b_{r}<\mathrm{g} & & \text { for } \mathrm{H} 0
\end{aligned}
$$

Where $\mathrm{H} 1$ represents that the final decision that has been made by the CR-BS, stating that the PR's signal is present in fi, and H0 represents the PR signal's absence. Number of CRs $\mathrm{g}$ that decides the presence of PR's signal at fi, determines the type of fusion rule at CR Base Station. When $g=1$ out of total G CRs, the fusion rule is "OR", the fusion rule is AND if only and if all $\mathrm{g}=\mathrm{G}$ CRs decides $\mathrm{H} 1$ case. Lastly when $1<\mathrm{g}<\mathrm{G}$ the "VOTING" fusion rule is applied.

In order to evaluate the cooperative spectrum sensing performance, we define three joint probabilities; the joint probability of detection, Qd(fi) the joint probability of false alarm Qf (fi), and the joint probability of missed detection
Qm (fi). The joint probability of detection can be written as given below:

Qd (fi) $=$ P( DECCOP $($ fi $)>=g / H 1)$

$\sum_{r=g}^{G}\left(\begin{array}{l}G \\ r\end{array}\right)\left[P\left(D E C(f i)>\frac{y}{H 1}\right)\right]^{r}\left[P\left(D E C(f i)<\frac{y}{H 1}\right]^{G-r}\right.$

$\sum_{r=g}^{G}\left(\begin{array}{l}G \\ r\end{array}\right) P d(f i)^{r}(1-P d(f i))^{G-r}$

And the joint probability of false alarm Qf(fi) can be written as given below:

$\mathrm{Qf}(\mathrm{fi})=\mathrm{P}($ DECCOP $(\mathrm{fi})>=\mathrm{g} / \mathrm{H} 0)$

$\sum_{=r=g}^{G}\left(\begin{array}{l}G \\ r\end{array}\right)\left[P\left(D E C(f i)>\frac{y}{H 0}\right)\right]^{r}\left[P\left(D E C(f i)<\frac{y}{H 0}\right]^{G-r}\right.$

$\sum_{r=g}^{G}\left(\begin{array}{l}G \\ r\end{array}\right) P f(f i)^{r}(1-P f(f i))^{G-r}$

Here we note that DEC(fi) here means the decision statistic of the used local sensing. So, finally the joint probability of missed detection can be written as follows:

$\begin{aligned} \mathrm{Qm}(\mathrm{fi})= & \mathrm{P}(\text { DECCOP }(\mathrm{fi})<\mathrm{g} / \mathrm{H} 1) \\ = & 1-\text { Qd }(\mathrm{fi})\end{aligned}$

Now the total error probability of the cooperative CR spectrum sensing is defined as given below [4]:

$$
\text { Qerror }=\text { Qm (fi) + Qf (fi) }
$$

\subsection{Local Spectrum Sensing Technique}

To examine the performance optimization, of the hard cooperative spectrum sensing, when local spectrum sensing techniques are used; the total error probability Qerror are evaluated at frequency bin fi, using Energy Detection. As we mentioned earlier in this paper, the different probabilities will be computed at a specific frequency bin fi. We have $G=10$ CRs co-operate the spectrum sensing decisions, at a CR-BS, in the CR's network. The local spectrum sensing techniques is ED. The local $\mathrm{SNR}=10 \mathrm{db}$, and $\mathrm{L}=10$ samples (i.e., OFDM blocks) are used locally for sensing. Here fig. 1 shows the total error probability ( Qerror) versus the chosen local threshold for $\mathrm{SNR}=10 \mathrm{db} \& \mathrm{~g}=5$ by theoretical method using ED technique. 
Fig. 2 shows the total error probability (Qerror) versus the chosen local threshold for SNR $=10 \mathrm{db} \& \mathrm{~g}=5$ by montecarlo simulation method using ED technique.

Here fig. 3 shows the total error probability (Qerror) versus the chosen local threshold for different number of $g$ out G CRs that controls the fusion rule in (13) using ED technique. If we compare the different curves that represent the total error for different numbers of $\mathrm{g}$ in Fig. 3, we observe, there are noticeable difference in the performance through using $\mathrm{g}=1$ to 10 as a $\mathrm{G}=10$ fusion rule. Here, $\mathrm{g}=10$ which represent "AND" fusion rule, gives high total error compared to the other curves; it is found that $\mathrm{g}=5$ gives the minimum total error (min Qerror) at the Same values of SNR and thresold. Hence, $g=5$ is the optimal fusion rule here (i.e., goptimal = 2).

$$
\mathrm{SNR}=10 \mathrm{db}, \mathrm{L}=10
$$

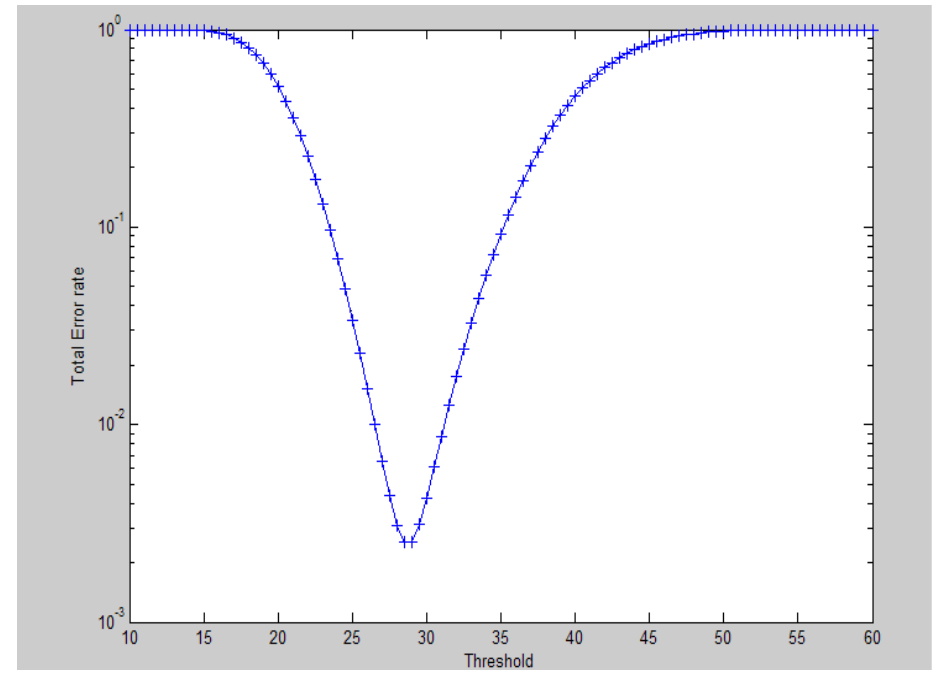

Fig. 1 Total error probability (Qerror ) for $g=5$ CRs versus local threshold when ED is used locally with SNR $=10 \mathrm{db}$ and $\mathrm{L}=10$ sensed samples used at each CR.(theoretical)

\subsection{Different Number of G CRS}

An interesting question now, is the goptimal that achieves min Qerror same when the number of total CRs is different? Table I shows the optimal fusion rule and min Q error when SNR is varied and the ED is used locally, with same number of the sensed samples (i.e. $\mathrm{L}=10$ ). The improvement in the performance by increasing the total number $G$ for different SNR at CRs at fixed L, is noticeable. For example, min Qerror $=0.2511$ when $\mathrm{SNR}=5 \mathrm{db}$ and $\mathrm{CRs}=4$ or 5 , and $\min =0.00251$ when SNR is increased to $10 \mathrm{db}$ and CRS $=5$. The increase in SNR causes decrease in the min Qerrorwith variation in number of CRs. Furthermore, for fixed SNR if the number of the total co-operated CRs, G, is increased above optimal then the Qerror is increased.
$\mathrm{SNR}=10 \mathrm{db}, \mathrm{L}=10$

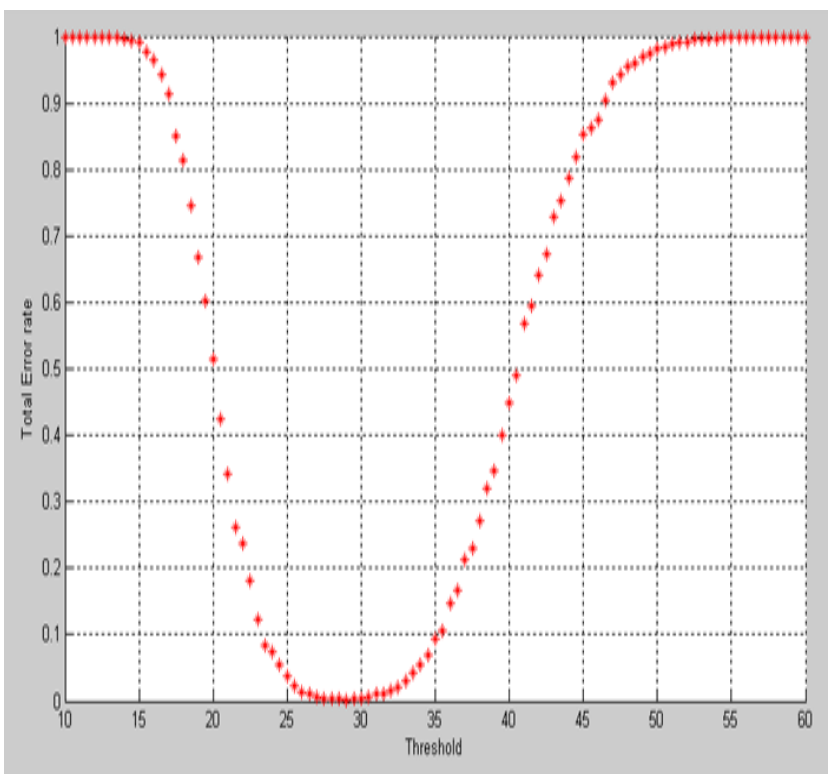

Fig. 2 Total error probability (Qerror ) for $\mathrm{g}=5 \mathrm{CRs}$ versus local threshold when ED is used locally with SNR $=10 \mathrm{db}$ and $\mathrm{L}=10$ sensed samples used at each CR.(monte-carlo simulation)

$$
\mathrm{SNR}=10 \mathrm{db}, \mathrm{L}=10
$$

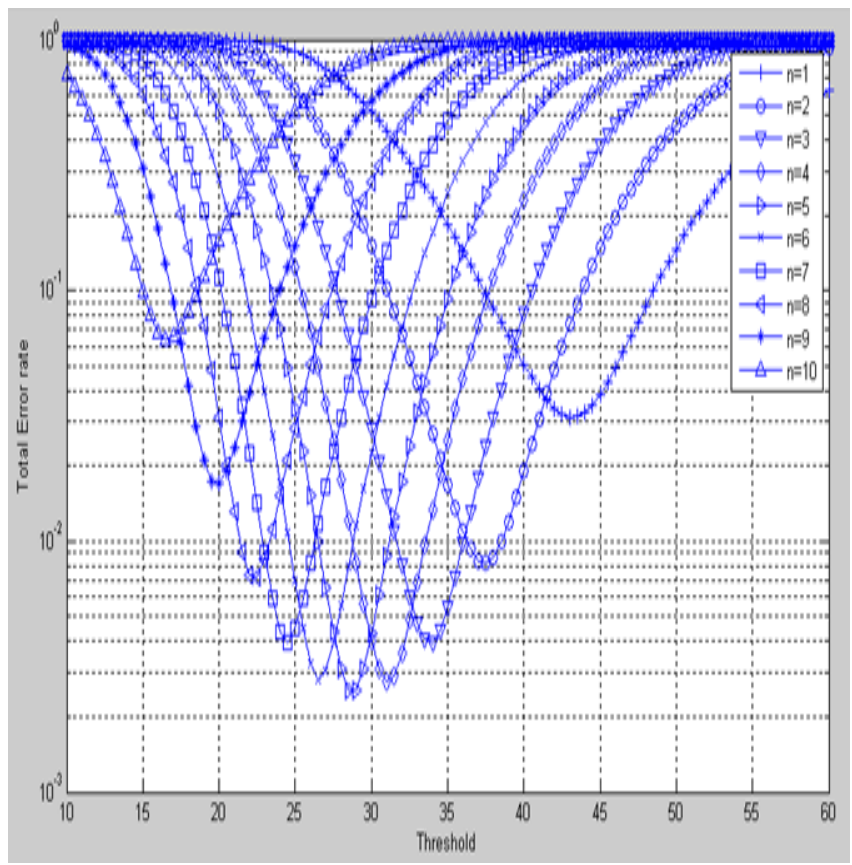

Fig. 3 Total error probability (Qerror) for g out of $\mathrm{G}=10 \mathrm{CRs}$ versus local threshold when $\mathrm{ED}$ is used locally with $\mathrm{SNR}=10$ $\mathrm{db}$ and $\mathrm{L}=10$ sensed samples used at each $\mathrm{CR}$ 
$\mathrm{SNR}=[5,10,13,17,18,20] \mathrm{db}, \mathrm{L}=10$
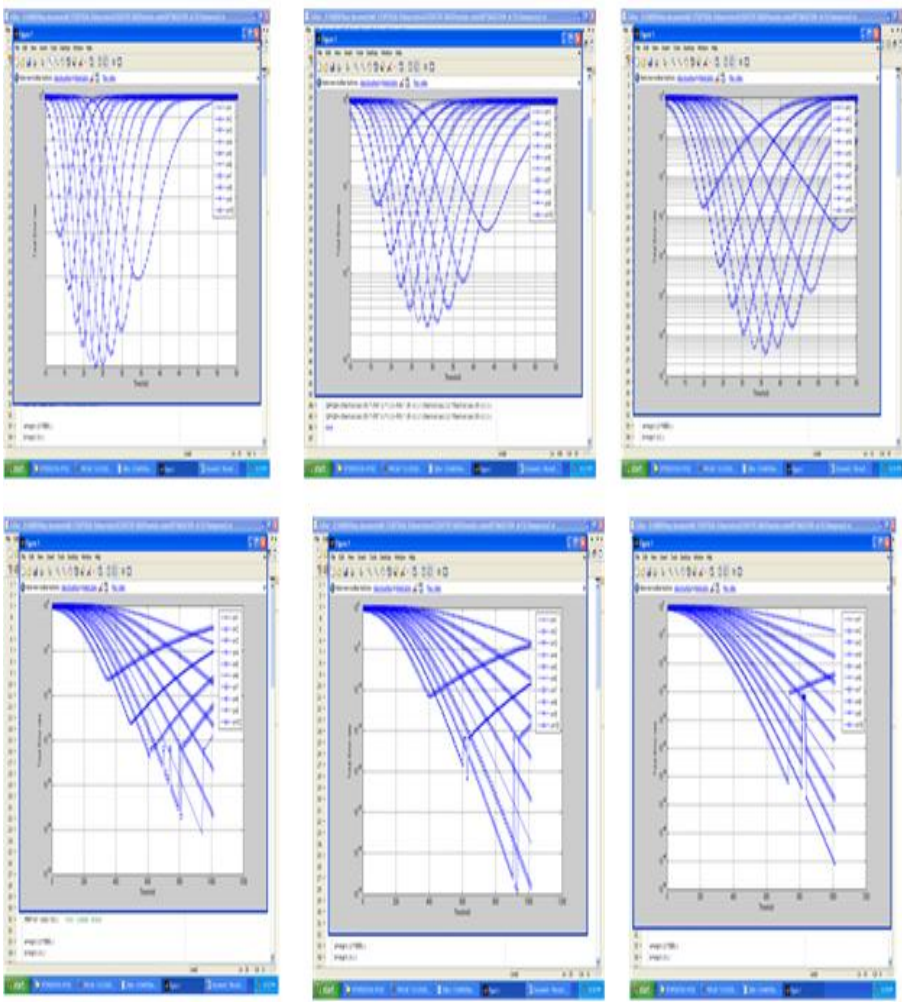

Fig. 4 Total error probability (Qerror) for g out of $\mathrm{G}=10$

CRs versus local threshold when ED is used locally with $\mathrm{SNR}=5,10,13,17,18,20 \mathrm{db}$ and $\mathrm{L}=10$ sensed samples used at each CR

In Fig. 3 we can see that for different SNRs we get different results regarding minimum Qerror and goptimal as we change the SNR error is decreased and also goptimal value is changing.

Table 1: Optimal number of g CRs for different SNR and its error level

\begin{tabular}{|l|l|l|}
\hline SNR in db & Error level & Number of cognitive radio user \\
\hline 5 & $10^{-0.6}$ & 4 or 5 \\
\hline 10 & $10^{-2.6}$ & 5 \\
\hline 13 & $10^{-6.4}$ & 5 or 6 \\
\hline 17 & $10^{-25}$ & 6 \\
\hline 18 & $10^{-34}$ & 8 \\
\hline 20 & $10^{-45}$ & 9 \\
\hline
\end{tabular}

Table I shows tabular form of fig. 3. It shows the variation in error level by changing SNR and respective Number of cognitive radio user.

\section{CONCLUSIONS}

This paper contributes to the cooperative spectrum sensing optimization by introducing an efficient optimization factor; the number of cognitive radio user, G. As at each CR module we may have different SNR, depending upon the value of SNR we have derived the total error rate for different $g$ (1 to $10)$. We have found the goptimal for each SNR. For SNR value $5,10,17,18,20$ we get goptimal as 4 or $5,5,5$ or 6,6 , 8,9 .

\section{REFERENCES}

[1]. O. A. Alghamdi, M. A. Abu-Rgheff, and M. Z. Ahmed, "MTM Parameters Optimization for 64-FFT Cognitive Radio Spectrum Sensing using Monte Carlo Simulation," in EMERGING 2010 : The Second International Conference on Emerging Network Intelligence, Florence-Italy, 2010, pp. 107113.

[2]. O. A. Alghamdi, M. Z. Ahmed, and M. A. Abu-Rgheff, "Probabilities of Detection and False Alarm in Multitaper Based Spectrum Sensing for Cognitive Radio Systems in AWGN," in The IEEE International Conference on Communication Systems (IEEE ICCS 2010) Singapore: IEEE, 2010

[3]. J. Shen, S. Liu, L. Zeng, G. Xie, J. Gao, and Y. Liu, "Optimisation of cooperative spectrum sensing in cognitive radio network," Communications, IET, vol. 3, pp. 1170-1178, 2009.

[4]. T. Yucek and H. Arslan, "A survey of spectrum sensing algorithms for cognitive radio applications," Communications Surveys \& Tutorials, IEEE, vol. 11, pp. 116-130, 2009.

[5]. Z. Wei, R. K. Mallik, and K. Ben Letaief, "Cooperative Spectrum Sensing Optimization in Cognitive Radio Networks," in Communications, 2008. ICC '08. IEEE International Conference on, 2008, pp. 3411-3415.

[6]. QinetiQ, "Cognitive Radio Technology - A Study for Ofcom - Summary Report," QINETIQ/06/00420, Issue 1.1, February 2007.

[7]. E. Peh and L. Ying-Chang, "Optimization for Cooperative Sensing in Cognitive Radio Networks," in Wireless Communications and Networking Conference, 2007.WCNC 2007. IEEE, 2007, pp. 27-32.

[8]. A. Ghasemi and E. S. Sousa, "Collaborative spectrum sensing for opportunistic access in fading environments, in New Frontiers in Dynamic Spectrum Access Networks, 2005. DySPAN 2005. 2005 First IEEE International Symposium on, 2005, pp. 131-136.

[9]. S. Haykin, "Cognitive radio: brain-empowered wireless communications," IEEE journal on selected areas in communications, vol. 23, pp. 201-220, 2005.

[10]. D. Cabric, S. M. Mishra, and R. W. Brodersen, "Implementation issues in spectrum sensing for cognitive radios," in Signals, Systems and Computers, 2004. Conference Record of the Thirty-Eighth Asilomar Conference on, 2004, pp. 772-776 Vol.1.

[11]. J. Mitola and G. Q. Maguire, "Cognitive radio: making software radios more personal," IEEE personal communications, vol. 6, pp. 13-18, 1999. 